\title{
Movement Strategies of Seed Predators as Determinants of Plant Recruitment Patterns
}

\author{
Lorenzo Mari, ${ }^{1, \star}$ Renato Casagrandi, ${ }^{1, \uparrow}$ Marino Gatto, ${ }^{1, \ddagger}$ Tal Avgar, ${ }^{2, \S}$ and Ran Nathan ${ }^{2, \|}$
}

1. Dipartimento di Elettronica e Informazione, Politecnico di Milano, Via Ponzio 34/5, 20133 Milano, Italy;

2. Movement Ecology Laboratory, Department of Evolution, Systematics and Ecology, Alexander Silberman Institute of Life Sciences, Hebrew University of Jerusalem, Jerusalem 91904, Israel

Submitted May 19, 2008; Accepted June 9, 2008;

Electronically published September 26, 2008

\begin{abstract}
Aвstract: Plant recruitment in nature exhibits several distinctive patterns ranging from hump shaped to monotonically decreasing with distance from the seed source. We investigate the role of postdispersal seed predation in shaping these patterns, introducing a new mechanistic model that explicitly accounts for the movement strategy used by seed eaters. The model consists of two partial differential equations describing the spatiotemporal dynamics of both seed and predator densities. The movement strategy is defined by how predators move in response to the different cues they can use to search for seeds. These cues may be seed density, seed intake, distance from the plant, density of conspecific foragers, or a mixture of these four. The model is able to reproduce all the basic plant recruitment patterns found in the field. We compare the results to those of the ideal free distribution (IFD) theory and show that hump-shaped plant recruitment patterns cannot be generated by IFD predators and, in general, by foragers that respond exclusively to seed density. These foragers can produce only nonincreasing patterns, the shapes of which are determined by the foragers' navigation capacities. In contrast, hump-shaped patterns can be produced by distance-responsive predators or by foragers that use conspecifics as a cue for seed abundance.
\end{abstract}

\footnotetext{
* Corresponding author; e-mail: mari@elet.polimi.it.

† E-mail: casagran@elet.polimi.it.

₹ E-mail: gatto@elet.polimi.it.

${ }^{\S}$ Present address: Department of Integrative Biology, University of Guelph, Guelph, Ontario N1G 2W1, Canada; e-mail: tavgar@uoguelph.ca.

" E-mail: rnathan@cc.huji.ac.il.

Am. Nat. 2008. Vol. 172, pp. 694-711. (c) 2008 by The University of Chicago. 0003-0147/2008/17205-50467\$15.00. All rights reserved.

DOI: $10.1086 / 591687$
}

Keywords: Janzen-Connell effect, postdispersal seed predation, foraging behavior, ideal free distribution, partial differential equations model.

Spatial patterns of plant recruitment are the result of fecundity, seed dispersal, and successive establishment processes (Howe and Smallwood 1982; Schupp and Fuentes 1995; Nathan and Muller-Landau 2000; Levin et al. 2003). Investigating the key processes underlying the emergence of these spatial patterns is important for understanding their causes and consequences at multiple levels of organization. Janzen (1970) and Connell (1971) pioneered this multilevel investigation and proposed the key role of spatial patterns of recruitment for the maintenance of high species richness in tropical forests and coral reefs. They hypothesized that the attraction of seed predators or seedling herbivores to the proximity of the propagule source generates spacing among conspecifics, thus permitting recruitment of other species and promoting coexistence.

The extensive research stimulated by these ideas has extended below the community level, exploring, for example, the consequences for population structure (Levin et al. 2003) and spread (Muller-Landau et al. 2003; Moorcroft et al. 2006), as well as the evolutionary causes at the individual level (Howe and Smallwood 1982). The key to understanding these multilevel phenomena is the analysis of basic plant-animal interactions, such as seed dispersal by animals, pre- and postdispersal seed predation by granivores, and seedling herbivory. The aim is to find the relationships between the physiological and behavioral characteristics of the consumers (which may affect their performances in detecting and depleting food resources) and the resulting spatial patterns of the plants on which they feed.

Although great attention has been devoted to the analysis of postdispersal seed predation in the field, a formal theory for space-dependent seed predation is still missing. To our knowledge, only two recent works (Nathan and Casagrandi 2004; Adler and Muller-Landau 2005) have used mathematical models to investigate the problem. Fol- 
lowing the approach of Janzen (1970), Nathan and Casagrandi (2004) assumed that predators respond to distance from the source and are displaced around it according to an exponentially decreasing function of distance. Their main finding is that distance-dependent predation alone is sufficient to generate all the different recruitment patterns found in nature. They also predicted several possible links between animal characteristics and plant recruitment patterns, most of which have been supported recently by a field study on harvester ants (Avgar et al. 2008). Instead, Adler and Muller-Landau (2005) focused on the effects of postdispersal seed predation on local biodiversity. Using a spatially explicit, individualbased model, they showed that the mean distances traveled by both seeds and predators are crucial to maintaining high species richness. The abundance of predators was dynamically linked to the density of adult trees, but the spatial distribution of predators was assumed to be fixed (with a negative exponential shape, as in Nathan and Casagrandi 2004).

Therefore, neither of the two models mentioned above explicitly incorporated predators' movements and the different movement strategies that foragers can adopt. The literature has considered the problem of consumers that can move from one location to another in response to different environmental cues (Kacelnik et al. 1992; Holmes et al. 1994; Sutherland et al. 2002; Dwyer and Morris 2006), but seed predation has received scant attention. For this case, the most cited cues are differences in seed density and distance from the seed source, although their relative influences on predators' activities is highly debated (density-dependent vs. distance-dependent seed predation; Janzen 1970; Hubbell 1980).

Here we offer improvement on the existing theory by abandoning the assumption that there is a predefined, time-invariant distribution of seed predators around the seed source. In the proposed model, the predator distribution can vary in time and space depending on the different movement strategies adopted by seed consumers. The predator-prey system is governed by two partial differential equations (PDEs): the first one is the Nathan and Casagrandi (2004) model, which describes spatiotemporal variations in the seed density; the second one describes the predator distribution and includes both random movement (represented by a diffusion term) and oriented movement (which is defined by the foraging strategy). To the best of our knowledge, the possible links between plant recruitment patterns and foraging strategies used by seed consumers have not yet been formally investigated.

This article is organized as follows. In "Plant Recruitment and Predation Patterns," we describe various plant recruitment patterns found in nature and provide an overview of the underlying seed predation mechanisms. Then, in "The Model," we introduce a new, spatially explicit model that couples the spatiotemporal dynamics of the food resources (dispersed seeds) with the spatiotemporal dynamics of seed predators. The model is used to investigate which plant recruitment patterns can be generated by predators adopting various movement strategies. Finally, we discuss the ecological implications of such behavioral characteristics of predators and suggest future directions of research.

\section{Plant Recruitment and Predation Patterns}

As spatial patterns of plant recruitment can be reshaped by postdispersal seed predation processes, different movement strategies are expected to produce different patterns of recruitment. Before formalizing a model to test this hypothesis, we provide an introduction to the variability of recruitment patterns found in nature and to the mechanisms that drive seed predation.

\section{The Variability of Natural Plant Recruitment Patterns}

Spatial patterns of plant recruitment vary enormously in nature, although they arise from patterns of seed dispersal that generally decline with distance from the plant (Willson 1993; Nathan and Muller-Landau 2000). One plausible reason for this discrepancy is the spatial variability of seed survival to predation. Specifically, different seed survival patterns can yield five qualitatively different recruitment types (McCanny 1985; Nathan and Casagrandi 2004). A thorough review of the literature (table 1 ) reveals empirical evidence for each of them. Note that the identification of recruitment patterns is neither simple nor univocal. In fact, the term "recruitment pattern" refers to a particular combination of dispersal, survivorship, and establishment curves rather than to the pattern described by the establishment (or seed survival) curve alone (Nathan and Casagrandi 2004).

The most cited pattern in the literature is the JanzenConnell (J-C) pattern (Janzen 1970; Connell 1971), which is characterized by a peak of established recruits at some distance from the seed source and by a monotonic increase in seed survival that is steeper than the decrease in seed dispersal (fig. 1A). This pattern was hypothesized to be driven by seed predators attracted to the seed source. This mechanism has been proposed as a plausible spacing mechanism capable of maintaining the high tree species diversity found in tropical forests (Janzen 1970). The J-C recruitment pattern has received only partial empirical support from studies focusing on single species (as reviewed in Hammond and Brown 1998; Wright 2002; Hyatt et al. 2003), but it has received strong support from anal- 
Table 1: Review of empirical evidence on seed and seedling recruitment patterns

\begin{tabular}{l} 
Recruitment pattern type \\
\hline Evidence for the Janzen-Connell recruitment pattern \\
Partial support for the Janzen-Connell model (but \\
either recruitment or survival has not been ex- \\
plicitly analyzed or reported)
\end{tabular}
plicitly analyzed or reported)

Evidence for the exact-compensation recruitment pattern

Evidence for the Hubbell recruitment pattern

Evidence for the invariant survival recruitment pattern

Evidence for the McCanny recruitment pattern

Rejection of the Janzen-Connell model (but reported data are insufficient to provide evidence for alternative patterns)
References

Hubbell 1979; Augspurger 1983a, 1983b, 1984; De Steven and Putz 1984; Itoh et al. 1997; Okuda et al. 1997; Barot et al. 1999; Nathan et al. 2000; Packer and Clay 2000; Wenny 2000; Gilbert et al. 2001; Wyatt and Silman 2004; Norghauer et al. 2006; Avgar et al. 2008

Janzen 1971a, 1972; Wilson and Janzen 1972; Howe and Primack 1975; Janzen et al. 1976; Denslow 1980; Clark and Clark 1984; Connell et al. 1984; Howe et al. 1985; Ramirez and Arroyo 1987; Hubbell et al. 1990; Traveset 1990; Condit et al. 1992; Forget 1994; Forget et al. 1994; Gilbert and De Steven 1996; Wills et al. 1997; Barone 2000; Grau 2000; Harms et al. 2000

Hubbell 1979; Itoh et al. 1997; Barot et al. 1999

Hubbell 1979; Wright 1983; McCanny and Cavers 1987; Forget 1992; Howe 1993; Okuda et al. 1997; Packer and Clay 2000

McCanny and Cavers 1987; Augspurger and Kitajima 1992; Condit et al. 1992; Forget 1992; Notman et al. 1996; Avgar et al. 2008; Boudreau and Lawes 2008

Guittet and Laberche 1974; Fleming and Heithaus 1981; McCanny and Cavers 1987; Augspurger and Kitajima 1992; Condit et al. 1992; Notman et al. 1996; Takeuchi and Nakashizuka 2007

Sork 1987; Schupp 1988; Forget 1989, 1993; Kitajima and Augspurger 1989; Hubbell et al. 1990; Burkey 1994; Terborgh and Wright 1994; Hart 1995

Note: Differences among patterns are described in the text.

yses of extensive plot data sets (Wills et al. 1997, 2006; Harms et al. 2000).

It must be remarked that there is an additional complication in finding evidence for the J-C pattern in the empirical literature. In fact, some studies (Augspurger 1983a; Clark and Clark 1984; Condit et al. 1992; Burkey 1994) report hump-shaped seed or seedling establishment patterns that may be not due to distance- or densitydependent predation but to the fact that the spatial arrangement of recruits around the parent plant has been evaluated as a radial distribution of seed numbers (rather than densities). This procedure can result in hump-shaped establishment patterns that are due to the geometry of seed dispersal (see again Augspurger 1983a; Burkey 1994). Therefore, hump-shaped establishment patterns of this kind cannot provide evidence for the J-C mechanism in the absence of further information.

The J-C pattern is not the only possible outcome of distance-dependent dispersal and postdispersal predation. In fact, these processes can also generate monotonically decreasing establishment curves if seed survival increases with distance at a lower rate than dispersal (Hubbell 1980) or declines with distance (McCanny 1985). The two resulting recruitment patterns have been termed, respectively, the Hubbell ( $\mathrm{H}$; fig. $1 B$ ) and the McCanny (McC; fig. 1C) patterns. The latter pattern in particular indicates predator satiation (Janzen $1971 b, 1976$ ) as hypothesized by Nathan and Casagrandi (2004; see also Takeuchi and Nakashizuka 2007). Intermediate between the J-C and the $\mathrm{H}$ patterns and the $\mathrm{H}$ and the $\mathrm{McC}$ patterns are two additional recruitment patterns named, respectively, exact compensation (EC) and invariant survival (IS). The EC pattern corresponds to seed survival increasing with distance at the same rate as the decrease of dispersal so that the plant recruitment is constant over space. The IS pattern is characterized by space-invariant seed survival.

\section{Seed Predation Mechanisms and the Ideal Free Distribution Theory}

Spatial patterns of seed survival to predation depend on the movement and consumption traits of granivores, 

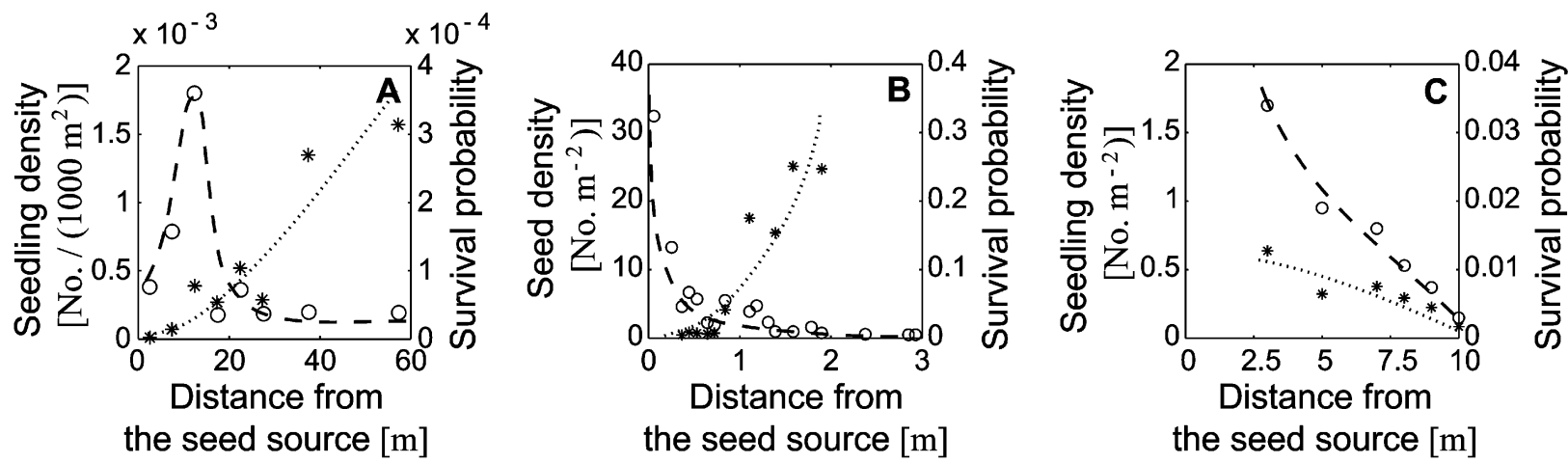

Figure 1: Examples of different recruitment patterns reported in the literature. A, Janzen-Connell pattern: seedlings of the pine Pinus halepensis (from Nathan et al. 2000); B, Hubbell pattern: seeds of the annual grass Panicum miliaceum (from McCanny and Cavers 1987); C, McCanny pattern: seedlings of the pine Pinus sylvestris (from Guittet and Laberche 1974). Open circles (left axis) and asterisks (right axis) correspond to seed establishment and survival, respectively. Dashed lines and dotted lines have been fitted by eye to aid the reader in clearly distinguishing the patterns.

which can be classified into those facilitating seed detection and intake (e.g., motion and navigation capacity) or constraining it (e.g., handling time and restricted home range). The ideal free distribution (IFD) theory (Fretwell and Lucas 1969) may help in defining the baseline model for the behavior of seed predators. According to the IFD theory, the resource is distributed in a set of discrete patches, while consumers are birds with no movement limitations, with complete information about the local distribution of the resources they feed on, perfect control of their own movement, and neither interference nor facilitation from conspecifics, competitors, or predators. Under these assumptions, IFD consumers should reach a distribution that perfectly matches the availability of their resources so that the per capita consumption rate is equalized over space. In other words, the consumers move among patches until the resource density in each location becomes equal. Therefore, IFD foragers are expected to generate the EC plant recruitment pattern.

Deviations from the IFD theory are the rule rather than the exception (Utne et al. 1993; Bautista et al. 1995; Cartar and Abrahams 1997; Hugie and Grand 1998; Shochat et al. 2002). In general, predators can deviate from the basic assumptions of the IFD model because of interference from conspecific foragers (Sutherland 1983), perceptual constraints (Abrahams 1986; Gray and Kennedy 1994), spatiotemporal variability of resources (Hakoyama 2003), and/or random motion (Jackson et al. 2004). Seed predators in particular can orient their movement using cues other than local resource abundance or intake rate; for instance, they can use distance from the seed source (Janzen 1970; Fragoso 1997) or conspecific density (Beauchamp et al. 1997; Koops and Abrahams 2003; Flaxman and Reeve 2006). Such deviations from the IFD can there- fore give rise to either resource overmatching or undermatching and, in turn, to patterns that are different from the EC (thus, the small number of references supporting EC in table 1 is not surprising). Specifically, the J-C pattern is generated by resource overmatching near the seed source (Freckleton and Lewis 2006) and by resource undermatching farther away. The McC pattern, on the other hand, is generated by opposing trends, that is, resource undermatching near the seed source and resource overmatching farther away (Nathan and Casagrandi 2004).

In this article we consider several types of deviations from the original IFD theory, all of which arise in the context of postdispersal seed predation. The most important deviation is the spatially explicit structure of the landscape, with a single plant acting as a seed source for the patches. Therefore, different spatial locations are characterized by different "carrying capacities" of seeds (sensu Fretwell and Lucas 1969). Consequently, the distance from the plant itself may be an important decision variable for predator movements, as it signals the potential availability of the resource. For instance, in the absence of other predators, a solitary forager should expect to find higher resource abundances closer to the source (e.g., Fragoso 1997). Although the actual seed density at a given location represents local information about resource abundance, the distance of a particular location from the seed source can serve as a cue for high resource densities that drive the movement of so-called distance-responsive seed predators (Janzen 1970, 1971a, 1972; Barone 2000; Wilson and Traveset 2000; Wyatt and Silman 2004). Also, the plant may represent an attraction for seed consumers independent of its role as a seed source, for instance, because it corresponds to the forager's central place of activity or 
provides shelter against predation (central-place foraging; e.g., Stephens and Krebs 1986).

Furthermore, we consider predators that are limited in movement and are thus characterized by finite movement speed. Also, we assume that a predator's movement is at least partially random. This seemingly marginal assumption could have nontrivial effects: on the one hand, diffusive movements have the potential to break the aggregation of seed predators in patches of high seed density, thus delaying patch depletion; on the other hand, partial randomness can interrupt sequences of highly correlated steps toward high-density patches, thus delaying patch detection.

As a final deviation from the IFD, we consider that seed predators can be sensitive to the density of conspecifics (Beauchamp et al. 1997; Koops and Abrahams 2003; Flaxman and Reeve 2006). In this way, we relax the basic assumption of no interaction among individuals that characterizes the IFD theory. Social foraging is a common strategy in many species that use spatially and temporally variable resources (e.g., Clode 1993), while interactions among predators can play an important role in the process of seed predation (Thompson et al. 1991; Serrano et al. 2001; Avgar et al. 2008). In some cases, the presence of conspecifics acting as seed predators in a given location may suggest that more food items can be found in less crowded locations (the local inhibition effect; see Goulson et al. 1998; Slaa et al. 2003). In other cases, it may indicate that an abundance of resources is locally available (the socalled local enhancement effect; see Turner 1964; Pöysä 1992; Buckley 1997; Slaa et al. 2003). Social foraging can also be used by predators to efficiently forage over large areas (Hancock et al. 2006) or to handle large food items (as in some species of ants; Hölldobler and Wilson 1990). Moreover, congregation (i.e., the attraction to conspecifics; see Beauchamp et al. 1997; Turchin 1998) can have causes other than foraging, such as mating and reduction of predation risk (Turchin and Kareiva 1989). Indeed, both positive and negative interactions among conspecific foragers represent deviations from the IFD theory (Beauchamp et al. 1997; Koops and Abrahams 2003; Flaxman and Reeve 2006).

\section{The Model}

Many spatially explicit PDE models for predator-prey dynamics have been proposed in the literature (as reviewed in Turchin 1998; Murray 2004). Simple advection-diffusion models (Skellam 1951; Patlak 1953) have often been used as reference models (see Okubo 1980; Okubo and Levin 2001), but they do not suffice with regard to the problem at hand. As we aim to explicitly link predators' movement strategies and plant recruitment patterns, we will consider that foragers can move following local cues that guide them in assessing the surrounding environment. In fact, a plethora of empirical evidence (Armsworth and Roughgarden 2005 and references therein) shows that oriented movements of predators can profoundly affect the resulting spatial patterns of abundances in the ecosystem.

In the biological literature, the movement of an organism in response to some external stimulus is generically termed "taxis" and is more specifically qualified as chemotaxis (Berg 1993), preytaxis (Kareiva and Odell 1987), or infotaxis (Vergassola et al. 2007) depending on the cues that are used (chemical concentrations, resource availability, or information, respectively). The few existing models accounting for taxis in consumer-resource problems involving complex organisms use space-dependent reproductive fitness of consumers as the principal factor influencing their local movements but do not explicitly model the environmental cues used for navigation (see, e.g., Ruxton and Rohani 1999; Armsworth and Roughgarden 2005). Interesting exceptions are the works by Grünbaum (1999), who defined a "response functional" used by predators as a proxy for environmental cues guiding them toward more favorable locations; by Farnsworth and Anderson (2001), who proposed a taxis-diffusion model for grazing that accounted for density-responsive predators; and by Cosner (2005), who derived a PDE model from which to discuss the mathematical basis of the IFD theory.

In this study, we assume that foragers can evaluate the quality of the surrounding landscape on the basis of the following information: (i) the local abundance of seeds or their current seed intake rate, (ii) the distance of their current location from the seed source, and (iii) the local density of their conspecifics. We aim to contrast IFD predators with non-IFD predators and, in particular, to test which information is key to obtaining qualitatively different recruitment patterns, that is, constant (EC), monotonically decreasing (H, IS, or McC), or hump-shaped (JC) establishment patterns.

We limit our attention here to the simple single-sourcesingle-predator case. Most real situations are certainly expected to be much more complex, being characterized by the presence of multiple seed sources with overlapping seed shadows, a mix of seeds from different species, and several species of both specialist and generalist seed predators. Nevertheless, despite its simplicity, our model is sufficient to describe real situations, such as desert ecosystems, which are usually characterized by the presence of sparse shrubs and a few taxa of specialized seed predators (e.g., Wilby and Shachak 2004). Furthermore, even in the highly diversified tropical forests, reproductive adult plants may be very rare and can thus be considered to be isolated seed sources (e.g., Muller-Landau et al. 2002). We 
consider a one-dimensional (1D) space because this allows us to derive analytical results concerning the patterns resulting from different movement strategies. However, the model can be easily extended to a two-dimensional (2D) space. In this case, the results can be obtained mainly through numerical techniques (L. Mari, R. Casagrandi, and M. Gatto, unpublished manuscript) but do not qualitatively differ from those in $1 \mathrm{D}$.

\section{Model Equations}

Let $S(x, t)$ and $N(x, t)$ indicate seed and predator densities, that is, the numbers of seeds or animals per unit area at location $x$ and time $t$. The location $x=0$ corresponds to the seed source. Because everything is symmetric, we consider only positive values of $x$ (distances from the parent plant). The basic equation for the spatiotemporal variation of seed density is identical to the 1D version of the Nathan and Casagrandi (2004) model, that is,

$$
\frac{\partial S(x, t)}{\partial t}=\Phi(x)-\mu S(x, t)-\psi(S) N(x, t),
$$

where $\Phi(x)$ represents the net flux of seeds at a distance from the source $x$ (the seed dispersal kernel; Willson 1993), $\mu$ is the seed mortality rate due to all causes other than predation, and $\psi(S)$ is the predators' functional response (Holling 1959). The mortality rate $\mu$ is kept constant here; that is, natural mortality is considered to be spatially homogeneous. This hypothesis is neither a priori valid nor necessary, but we use it to avoid potential confounding effects. In fact, higher natural mortality in the proximity of the seed source (due, for instance, to plant-soil negative feedbacks; Mazzoleni et al. 2007; Bonanomi et al. 2008) can surely influence seed establishment patterns. We make no specific assumptions on the functional forms of $\Phi(x)$ and $\psi(S)$ except for requiring that some obvious biological constraints be satisfied. Specifically, we assume that the dispersal kernel $\Phi(x)$ does not increase with distance from the source and vanishes for very large values of $x$, while the predators' intake rate $\psi(S)$ is assumed to be vanishing at $S=0$, nondecreasing in $S$, and possibly, but not necessarily, saturating at high seed densities. We also remark that the seeds that remain on the ground at the end of the dispersal and predation season represent only potential recruits because there might be mechanisms other than seed predation and natural mortality (possibly occurring after postdispersal seed predation has taken place) that could modify the density and distribution of seeds or seedlings (see the relevant discussion in Nathan and Casagrandi 2004).

As for predator density, we derive the dynamical equation that describes the movements of predators combining random search for seeds (diffusion) and directional attraction toward adjacent locations with high environmental quality (nonlinear advection). The resulting equation, the derivation of which is simple and can be found, for instance, in Cosner (2005), is

$$
\begin{aligned}
\frac{\partial N(x, t)}{\partial t} & =-\frac{\partial J(x, t)}{\partial x} \\
& =-\frac{\partial}{\partial x}\left[\varepsilon \frac{\partial f(x, t)}{\partial x} N(x, t)-\delta \frac{\partial N(x, t)}{\partial x}\right]
\end{aligned}
$$

where $J(x, t)$ represents the net flux of predators at location $x$ and time $t$ and $\varepsilon$ and $\delta$ are the taxis and the pure diffusion coefficients, respectively. The function $f(x, t)$, termed here as "habitat suitability" as in Fretwell and Lucas (1969), is an indicator of the attractiveness of location $x$ at time $t$. We express the habitat suitability function (an adimensional variable) in general terms as

$$
f(x, t)=F(x, S(x, t), N(x, t)) .
$$

According to the basic idea of the IFD theory elaborated on by Fretwell and Lucas (1969) and of the predators' aggregational response proposed by Hassell and May (1974), locations with high resource densities are, of course, attractive to seed predators. Therefore, without loss of generality, we can assume that $F$ does not decrease with increasing values of seed density, that is, that $\partial F / \partial S \geq 0$.

As for the dependence of $F$ on distance, it must be remarked that the plant may be seen as a seed source and/ or a focal activity place (e.g., because predators nest in the proximity of the plant). In the first case, distance can serve as a cue for high resource densities (distance-dependent predation; e.g., Janzen 1970). In the second case (centralplace foraging; e.g., Stephens and Krebs 1986), locations close to the plant are preferred, for instance, because they are "less expensive" than those farther away from an energy expenditure viewpoint. The fact that distance from the focal place should be considered in models aimed at estimating the foraging ranges of central-place consumers has been clearly pointed out in previous studies, such as those of Rosenberg and McKelvey (1999) and Nemeth et al. (2005). Without loss of generality, however, we can expect that ceteris paribus locations farther from the seed source cannot be more attractive than the closer ones, and we can safely assume that $F$ does not increase with distance from the source, that is, that $\partial F / \partial x \leq 0$.

The function $F$ can also depend on $N$. In fact, the presence of conspecific predators can be an important cue for resource availability (local enhancement; Turner 1964). Cooperation with conspecifics can be used by invertebrates (e.g., Slaa et al. 2003) and by vertebrates (Pöysä 1992; 
Buckley 1997) to efficiently forage over space. On the other hand, it has sometimes been reported that predators tend to avoid conspecifics (local inhibition; e.g., Goulson et al. 1998; Slaa et al. 2003). Therefore, $F$ can be either nondecreasing with respect to the local density of foragers $(\partial F / \partial N \geq 0)$ if there is enhancement or decreasing $(\partial F / \partial N<0)$ if there is inhibition.

The taxis coefficient $\varepsilon$ relates the gradient of the habitat suitability $f(x, t)$ to the corresponding movement speed of individuals (Segel 1977). The diffusion coefficient $\delta$ describes the magnitude of the random movement of predators (according to an uncorrelated random walk; Turchin 1998; Okubo and Levin 2001). Because the habitat suitability $f$ is dimensionless, both $\varepsilon$ and $\delta$ are measured as areas per unit time and, therefore, the $\varepsilon / \delta$ ratio is also dimensionless. It represents a measure of the predators' navigation capacity (sensu Nathan et al. 2008), namely, the ability to move toward targeted cues as opposed to random movement. We note that navigation capacity is by far the less explored component of animal movement in both empirical and theoretical studies (Holyoak et al. 2008).

Equation (2) does not account for the demography of predators but describes only their movement. In fact, we assume that, during the period of seed availability, the predators do not reproduce and do not suffer from any mortality. Therefore, we have $\int_{0}^{\infty} N(x, t) d x=\beta$, where $\beta$ is the total number of predators over positive values of $x$. Of course, this would not be true if the predator generation length were comparable to the length of the seed release period.

\section{Calculating Plant Recruitment Patterns}

Seed survival and establishment patterns can be obtained as stable stationary solutions (temporal steady states) of our PDE model (eqq. [1], [2]). This is correct, provided that recruitment patterns are observed after a sufficiently long time following the release of seeds from the parent plant. To find the stationary solutions, we have to nullify the time derivative in equations (1) and (2) and impose a zero-flux condition for predators (i.e., $\lim _{x \rightarrow \infty} J(x)=0$ ). This last condition is because of the assumption of finite seed predator population abundance. Technically, this is equivalent to imposing $\lim _{x \rightarrow \infty} N(x)=0$ and $\lim _{x \rightarrow \infty} \partial N(x) / \partial x=0$, that is, to solving a boundary value problem. Thus, the steady states $\bar{S}(x)$ and $\bar{N}(x)$ of equations (1) and (2) can be found as solutions of the following system:

$$
\left\{\begin{array}{l}
\bar{N}(x)=\frac{\Phi(x)-\mu \bar{S}(x)}{\psi(\bar{S}(x))} \\
\bar{J}(x)=\varepsilon \frac{\partial f(x)}{\partial x} \bar{N}(x)-\delta \frac{\partial \bar{N}(x)}{\partial x}=0
\end{array}\right.
$$

By substituting the first of equation (3) into the second and by suitably differentiating, the above system reduces to a single first-order ordinary differential equation in space for $\bar{S}(x)$. This equation has an infinite number of solutions, among which we will identify the one satisfying the constraint on the total number of predators $\int_{0}^{\infty} \bar{N}(x) d x=\beta$.

Our main goal is to determine which ecological conditions determine different establishment patterns $\bar{S}(x)$ : constant (EC), hump shaped (J-C), or monotonically decreasing $(\mathrm{H}$, IS, or McC). We identify the transition between these families of patterns by looking at the sign of the derivative of the seed establishment curve at the seed source. In particular, the EC pattern is conceptually identified by $\partial \bar{S}(x) /\left.\partial x\right|_{x=0}=0$ (numerically we consider $0 \leq$ $\partial \bar{S}(x) /\left.\partial x\right|_{x=0} \leq \chi$, with $\chi$ being a suitably small positive number), whereas the J-C pattern is characterized by an increasing establishment curve near the seed source, that is, $\partial \bar{S}(x) /\left.\partial x\right|_{x=0}>0$ (numerically, $\partial \bar{S}(x) /\left.\partial x\right|_{x=0}>\chi$ ) and the $\mathrm{H}, \mathrm{IS}$, or McC pattern by a decreasing establishment curve, that is, $\partial \bar{S}(x) /\left.\partial x\right|_{x=0}<0$. The discrimination among the $\mathrm{H}$, IS, and McC patterns depends on the shape of the probability that seeds escape predation, $\bar{P}(x)$, that is,

$$
\bar{P}(x)=\frac{\bar{S}(x)}{\Phi(x) / \mu}=\mu \frac{\bar{S}(x)}{\Phi(x)} .
$$

There is a slight complication, because $\bar{P}(x)$ is in any case a function increasing up to 1 for very large distances from the source. As detailed in Nathan and Casagrandi (2004), it is necessary to establish whether, for short to medium distances from the source, $\bar{P}(x)$ is increasing $(\mathrm{H})$, constant (IS), or decreasing (McC).

\section{Results}

In order to examine the effects of IFD and non-IFD predators on seed establishment patterns, we separately study four different classes of the habitat suitability function $f$ that correspond to four different movement strategies. The habitat suitability $f$ can depend on (i) seed density (or intake rate) only, (ii) distance from the plant, (iii) both distance from the plant and seed density, or (iv) both seed density and conspecific abundance. 


\section{Density-Responsive Predators and IFD Movement Strategies}

The simplest case of exclusively density-dependent foraging is modeled by a habitat suitability of the form

$$
f(x, t)=g(S(x, t))
$$

where $g(S)$ is a nondecreasing function of $S$. Many choices for $g(S)$ are possible, of course, but the following two are the most natural: either $g$ is linear $(g=b S$, where the positive parameter $b$ represents the animals' sensitivity to variations of resource abundance) or $g=\psi(S)$, when foragers are more sensitive to their actual food intake rate rather than the resource abundance per se. In any case, equation (3) for obtaining the stationary spatial distributions of seeds, $\bar{S}(x)$, and animals, $\bar{N}(x)$, translates into the following ordinary differential equation:

$$
\frac{\partial \bar{S}(x)}{\partial x}=\frac{\frac{\partial \Phi(x)}{\partial x}}{\frac{\varepsilon}{\delta} \frac{\partial g(\bar{S}(x))}{\partial \bar{S}(x)} \psi(\bar{S}(x)) \bar{N}(x)+\mu+\frac{\partial \psi(\bar{S}(x))}{\partial \bar{S}(x)} \bar{N}(x)}
$$

Because $\partial \bar{S}(x) / \partial x$ is the ratio between a nonpositive numerator and a positive denominator (recall that the dispersal kernel $\Phi(x)$ does not increase with distance, whereas both $\psi(S)$ and $g(S)$ do not decrease with seed density), the spatial derivative of the stationary establishment curve cannot be positive, regardless of the values assumed by the parameters and for any location $x$. Thus, $\bar{S}(x)$ is nonincreasing with $x$. This has the important implication that the J-C pattern cannot be generated by purely density-responsive predators.

Equation (4) allows us to understand under which conditions the EC pattern, corresponding to IFD predators, can emerge. It is apparent that the spatial derivative of the establishment curve in the proximity of the seed source tends to vanish in the limit of a high $\varepsilon / \delta$ ratio, even in cases of rapidly decreasing seed dispersal kernels. In fact, high navigation capacity corresponds not to predators that move randomly but to those that completely control their movement by solely responding to higher resource concentration. Note that these characteristics perfectly fit the description of IFD predators.

To better clarify this result, we have performed some numerical experiments. We have assumed specific functional forms for the seed dispersal kernel, the predators' functional response, and the habitat suitability $f(g(S))$. As for the dispersal kernel, we have used an exponential function of the form

$$
\Phi(x)=\frac{\alpha}{D} \exp \left(-\frac{x}{D}\right),
$$

in which $\alpha$ and $D$ represent the total seed output and the mean distance traveled by dispersed seeds, respectively. As for seed predation, we have assumed a Type II functional response (Holling 1959),

$$
\psi(S)=\frac{a S}{1+a T_{\mathrm{h}} S},
$$

where $\alpha$ and $T_{\mathrm{h}}$ represent the predator searching efficiency and handling time, respectively. The function $g(S)$ has been assumed to linearly depend on $S$, that is, $g(S)=b S$. From a technical viewpoint, integrating the ordinary differential equations derived from the full PDE model in order to evaluate the stationary solutions requires very accurate numerical methods. In particular, we have used an explicit eighth-order Runge-Kutta method with automatic control of the step size (Prince and Dorman 1981).

For low values of the $\varepsilon / \delta$ ratio (fig. $2 A$ ), seed predation results in a monotonically decreasing $\mathrm{H}$ pattern. Therefore, $\mathrm{H}$ patterns may represent the outcome of density-dependent seed predation exerted by animals with limited navigation capacity. As expected, when the level of navigation capacity $\varepsilon / \delta$ is increased (fig. $2 B, 2 C$ ), the resulting recruitment pattern is increasingly similar to the EC pattern. This condition corresponds to predators with very high orientation capabilities and, consequently, might not be very common in nature (table 1 ).

The corresponding stationary distribution of seed predators (fig. 2D) is very interesting, because there are no predators located farther than a critical distance from the seed source. Therefore, IFD predators may aggregate even if the resource density gradient is continuous. We emphasize that the spatial distribution of predators is not imposed a priori but results from the application of our model. If the landscape included several sparse seed sources (Muller-Landau et al. 2002), IFD predators would display a clumped distribution because they would congregate around each plant. Also notice that the predators' seed intake is constant over space (fig. $2 D$ ), as predicted by the classical IFD theory.

\section{Non-IFD Movement Strategies: Purely Distance-Responsive Predators}

The simplest mathematical way in which to account for purely distance-dependent predation (sensu Janzen 1970) is to consider the following linear expression for the habitat suitability function: 

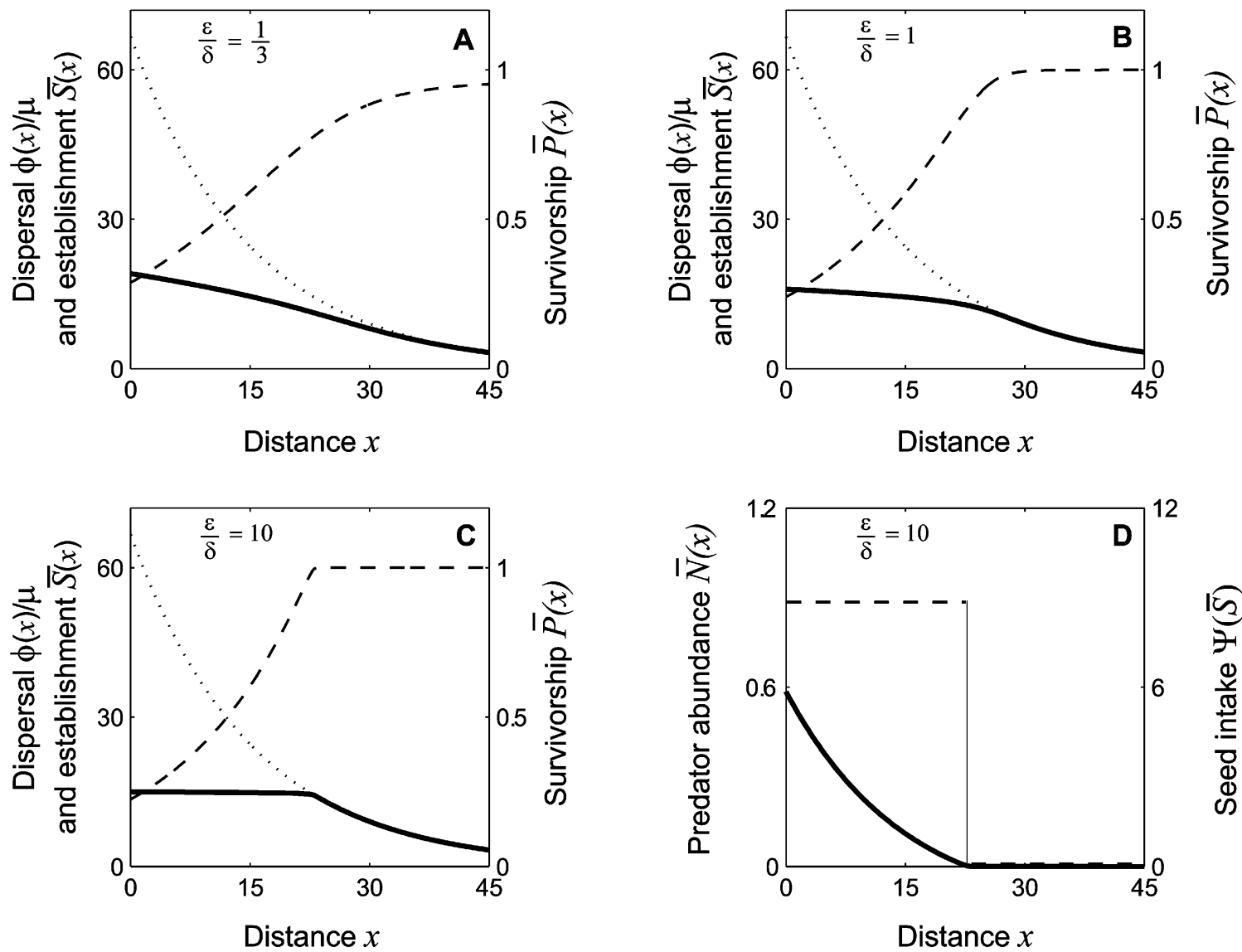

Figure 2: Patterns generated by the stationary solutions of equations (1) and (2) in the case of purely density-dependent predation $f(x, t)=$ $b S(x, t)$. Seed dispersal is described as recruitment in absence of predation $(\Phi(x) / \mu$; dotted line, left axis). Patterns of seed establishment $(\bar{S}(x)$; solid line, left axis) and the probability that seeds escape predation $(\bar{P}(x)$; dashed line, right axis) are obtained by setting $\varepsilon / \delta$ to three different values, that is, $1 / 3(A), 1(B)$, and $10(C)$. D shows the predator distribution $(\bar{N}(x)$; solid line, left axis) and per capita intake rate $(\psi(\bar{S})$; dashed line, right axis) with $\varepsilon / \delta=10$. Other parameter values are $\alpha=100, D=15, \mu=0.1, \alpha=5, T_{k}=0.1, \beta=5$, and $b=1$.

$$
f(x, t)=h(x)=-\tau x+\text { constant }
$$

where the positive parameter $\tau$ is a measure of the attractiveness of the parent plant. Although some authors suggest that response to distance might be nonlinear (e.g., Naef-Daenzer 2000), data confirming the simpler hypothesis made above have already been reported in the literature (Andersson 1981). However, we remark that the following discussion on the qualitative shape of the resulting establishment patterns is valid for any nondecreasing function of distance $h(x)$, either linear or nonlinear.

It is a matter of algebraic calculation (see again eq. [3]) to verify that the stationary distributions of seeds over space must, in this case, solve the following ordinary differential equation:

$$
\frac{\partial \bar{S}(x)}{\partial x}=\frac{\frac{\partial \Phi(x)}{\partial x}+\frac{\varepsilon}{\delta} \tau(\Phi(x)-\mu \bar{S}(x))}{\mu+\frac{\partial \psi(\bar{S}(x))}{\partial \bar{S}(x)} \bar{N}(x)} .
$$

Because the denominator of this expression is always positive, the sign of the spatial derivative of the seed establishment depends only on the sign of the numerator. In particular, a positive derivative of the establishment curve close to the plant is found if

$$
\bar{S}(0)<\frac{1}{\mu}\left(\Phi(0)+\left.\frac{\delta}{\varepsilon \tau} \frac{\partial \Phi(x)}{\partial x}\right|_{x=0}\right) .
$$

Therefore, purely distance-dependent predation has the power of generating hump-shaped (J-C) patterns. If we 
A
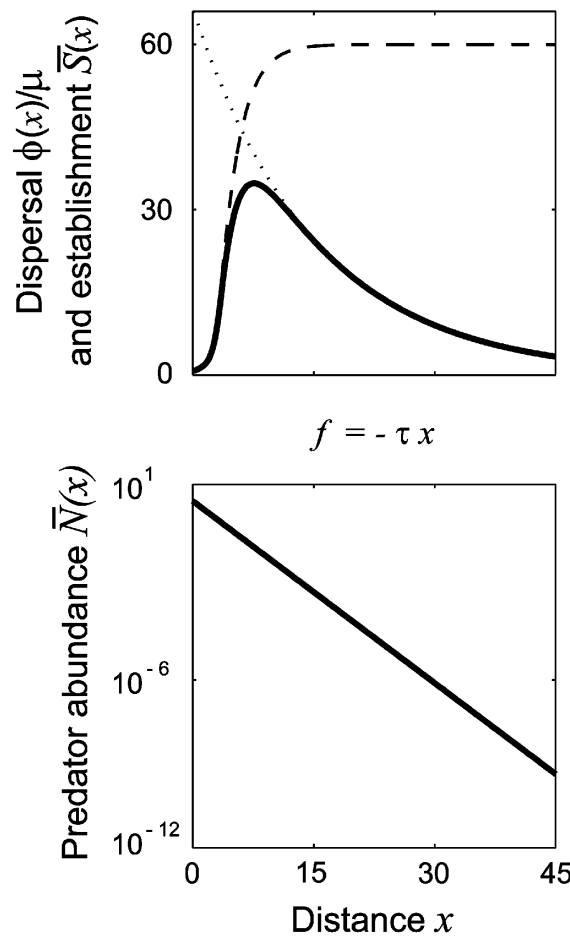

B

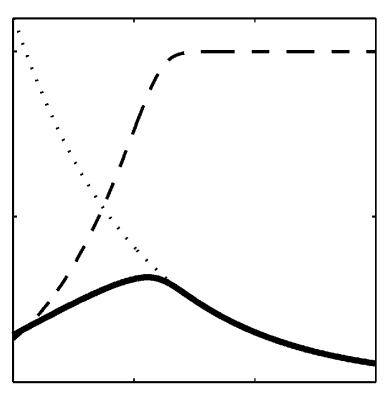

$f=b S-\tau x$

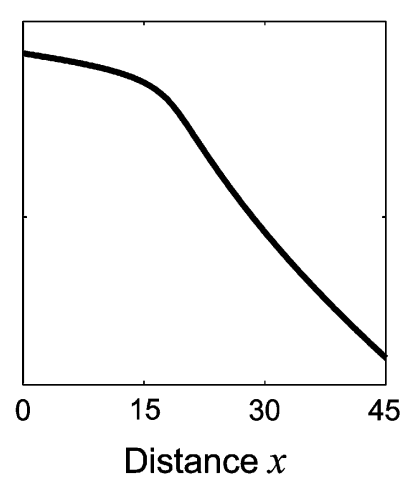

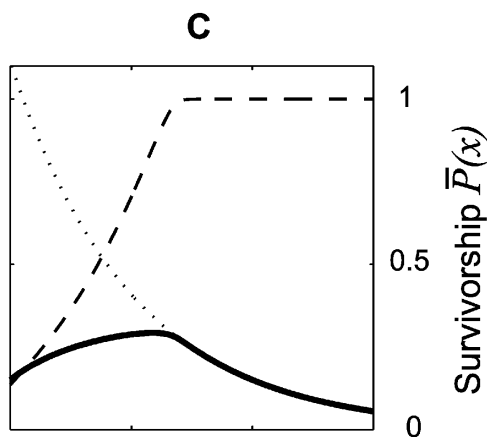

0

$$
f=b S+c N
$$

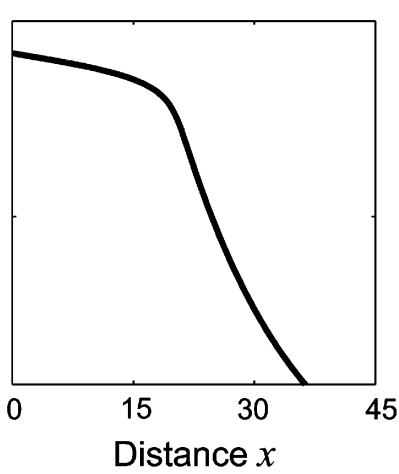

Figure 3: Janzen-Connell patterns (top) and their respective predator distributions (bottom; semi-log scale) generated by equations (1) and (2) with different habitat suitability functions. Top, solid lines and dotted lines indicate establishment patterns $(\bar{S}(x)$; left axis) and seed dispersal $(\Phi(x) / \mu$; left axis), whereas dashed lines indicate the probability that seeds escape predation $(\bar{P}(x)$; right axis). $A, f(x, t)=-\tau x$ with $\tau=1$ and $\varepsilon / \delta=1 / 2$. $B, f(x, t)=b S(x, t)-\tau x$ with $b=1, \tau=1$, and $\varepsilon / \delta=1 / 2 . C, f(x, t)=b S(x, t)+c N(x, t)$ with $b=1, c=15$, and $\varepsilon / \delta=2$. Unspecified parameters are equal to those specified in figure 2 .

use the negative exponential kernel for seed dispersal introduced above, we find that establishment patterns are hump shaped if

$$
\bar{S}(0)<\frac{\Phi(0)}{\mu}\left(1-\frac{\delta}{\varepsilon \tau D}\right) .
$$

On the other hand, the condition $J(x)=0$ provides (see eq. [3])

$$
\bar{N}(x)=\bar{N}(0) \exp \left(-\frac{\varepsilon \tau}{\delta} x\right)=\frac{\beta}{q} \exp \left(-\frac{x}{q}\right)
$$

where $q=\delta / \varepsilon \tau$ is the average distance of predators from the seed source. Because

$$
\bar{N}(0)=\frac{\beta}{q}=\frac{\Phi(0)-\mu \bar{S}(0)}{\psi(\bar{S}(0))},
$$

$\bar{S}(0)$ is a decreasing function of the total number of pred- ators $\beta$. Consequently, equation (5) can be satisfied for large $\beta$ and, in turn, $\mathrm{J}$-C patterns are possible if there is a sufficiently large number of seed predators around the source. It must be remarked that equation (5) is equivalent to the criterion for the identification of J-C patterns proposed by Nathan and Casagrandi (2004). The advantage of the present analysis is that it explicitly links the average distance $q$ to the predators' movement strategy.

Note that, at equilibrium, purely distance-dependent predators are spatially displaced around the seed source according to an exponential distribution. This result is completely independent of the type of functional response that is actually considered. Therefore, the a priori assumption of negative exponential distribution of predators around the seed source made by previous models (Nathan and Casagrandi 2004; Adler and Muller-Landau 2005) implicitly refers to purely distance-dependent predation and, in particular, to a linear decrease of habitat suitability with distance from the plant.

Figure $3 A$ shows the stationary spatial distribution of seeds and predators corresponding to a typical J-C pattern 
as generated by our model with distance-dependent predation only. Parameter settings that are different from those used in figure $3 A$ and that violate equation (5) can lead to either constant (EC) or monotonically decreasing (H, IS, or McC) patterns. Distance-dependent foraging can in fact explain all the basic families of recruitment patterns, as already shown by Nathan and Casagrandi (2004).

\section{Mixed Movement Strategies Depending on Both Seed Density and Distance from the Source}

The cases examined above are two extremes of a wide spectrum of possibilities, because both distance and density can serve as important environmental cues driving predators' movements. It is thus interesting to explore what kind of patterns can emerge if the habitat suitability takes a mixed form, such as

$$
f(x, t)=b S(x, t)-\tau x+\text { constant. }
$$

Note that the choice of using a linear combination of $S$ and $x$ in the formalization of $f$ is just instrumental and is aimed at simplicity. Any function $F$ satisfying the hypotheses introduced in the previous section would produce the same qualitative results. Because the habitat suitability function now contains both density- and distanceresponsive predation as two subcases, all the patterns previously classified can obviously be obtained. However, predator distributions associated with hump-shaped establishment patterns obtained with the mixed habitat suitability function can be quite different from the patterns corresponding to the case of pure distance dependence. In particular, most predators can gather within a critical distance from the plant (fig. 3B). This pattern is similar (although not identical) to the clumping in the EC case (fig. 2C, 2D). Note that we can identify a threshold in the predators' distribution at the distance where the graph of $\log \bar{N}(x)$ changes its slope. Whereas J-C patterns for the mixed habitat suitability can differ from those of purely distance-responsive predators, the monotonically decreasing establishment patterns are very similar to those obtained with strict dependence on distance. In fact, for the large range of parameter values we have explored, the predators' distribution is quite close to a negative exponential distribution (not shown here).

The resulting patterns for the mixed movement strategies crucially depend on two parameters: the ratio $\varepsilon / \delta$, which describes the predators' navigation ability, and the ratio $\tau / b$, which defines the relative importance of the density-responsive versus distance-responsive components of mixed movement strategies. Unfortunately, this quantity is not a pure number because it has the units of a seed density divided by a distance (remember that habitat suitability is dimensionless). Therefore, we have decided to perform the analysis by setting both $\tau$ and $\delta$ to unity and letting $b$ and $\varepsilon$ vary over four orders of magnitude (from $10^{-2}$ to $10^{2}$ ). This range of variation turned out to be sufficient to capture all possible patterns.

Figure 4 shows that, if the ratio $\tau / b$ is very small (i.e., if predators are mainly density responsive), no J-C plant recruitment patterns can become established. Figure 4 shows that there is a threshold of the navigation capacity $\varepsilon / \delta$ below which the random, diffusive component of movement is too significant for seed predation to result in EC patterns. Only if the ratio $\tau / b$ is sufficiently highthat is, if predators are more distance responsive than density responsive-is the $\mathrm{McC}$ pattern obtained. The superimposed dotted curves are contour lines of the function $q / D$, that is, the ratio between the average distance of predators from the seed source and the average distance traveled by dispersed seeds. We remark that, while $D$ is a parameter of the model, $q$ is not and must be evaluated ex post from the model parameters and solutions because it is the result of the behavioral response of seed predators. It is interesting and important to note that, when $q$ and $D$ have comparable values, the emergent pattern is of the $\mathrm{H}$ type, whereas a McC pattern emerges when $q$ is much

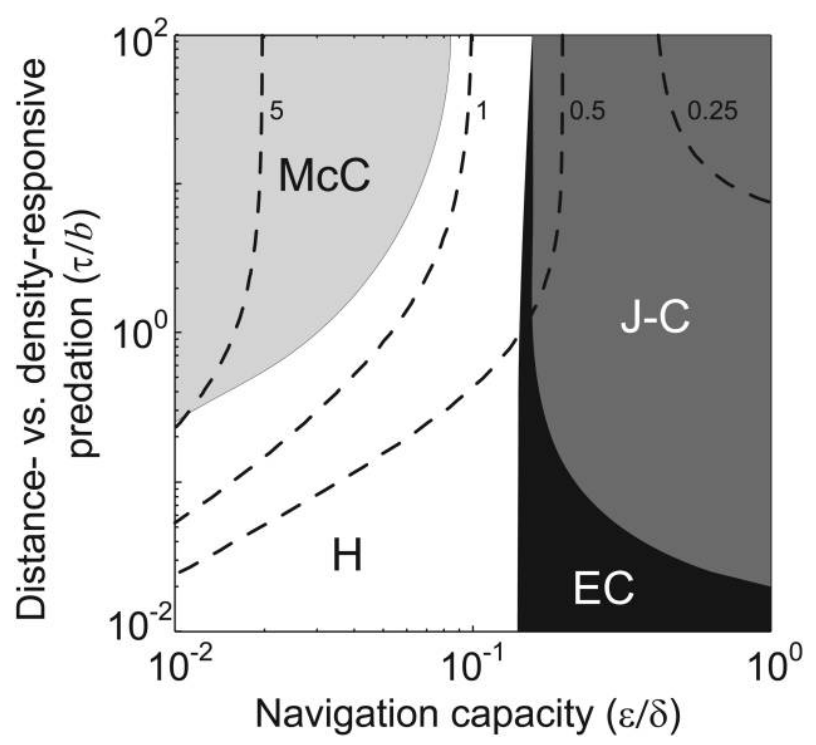

Figure 4: Recruitment patterns generated by models (1) and (2) with habitat suitability $f(x, t)=b S(x, t)-\tau x$ as functions of the navigation capacity $\varepsilon / \delta$ and the distance- versus density-responsive predation ratio $\tau / b$. Parametric regions with qualitatively similar patterns are identified by different color shadings. In particular, white = Hubbell $(\mathrm{H})$, light gray = McCanny $(\mathrm{McC})$, dark gray = Janzen-Connell $(\mathrm{J}-\mathrm{C})$, and black $=$ exact compensation (EC). Dashed curves $=$ isolines of $q / D$ in parameter space $(\varepsilon / \delta, \tau / b)$. Parameters are equal to those specified in figure 2 , except $D=10$. 
larger than D. Hump-shaped patterns can be obtained in cases in which predators are significantly distance responsive (high $\tau / b$ ) and capable of navigated movement (high $\varepsilon / \delta)$. Correspondingly, predators are distributed over space with a smaller mean distance from the plant than are the dispersed seeds $(q<D)$.

Therefore, as has already been pointed out by Nathan and Casagrandi (2004), the ratio between the average distance of predators and that of dispersed seeds from the plant is crucially linked to the emergence of different recruitment patterns. The two distances are of great importance because they can be directly estimated in the field. It is therefore interesting to report the model behavior in the space $(q, D)$. Figure 5 shows the partitioning of this space into the regions corresponding to $\mathrm{J}-\mathrm{C}, \mathrm{EC}, \mathrm{H}$, or $\mathrm{McC}$ patterns for different values of the parameter $b$ (i.e., for different sensitivities to seed density in mixed movement strategies). Figure 5 is obtained by finding the resulting patterns and the corresponding average predator distance for different values of $D$ and $\varepsilon / \delta$ and by displaying the patterns as functions of $q$ and $D$.

Figure 5 confirms the findings of figure 4 and shows that parameter $b$ plays an important role in determining recruitment patterns. In fact, increasing the sensitivity $b$ of predators to seed density generally determines an enlargement of the $\mathrm{H}$ pattern region in the parameter space $(q, D)$. This can be expected, because exclusively densityresponsive predators cannot generate J-C patterns, as we have already formally demonstrated. Moreover, at least for some parametric combinations, the backward-bending shape of the curves separating the $H$ patterns from the $\mathrm{McC}$ patterns produces an interesting phenomenon: for a given mean distance of predators from the seed source (i.e., for a given spatial distribution of foragers), McC patterns are determined by intermediate values of the mean distance $D$ traveled by seeds, whereas either low or high values of $D$ result in $\mathrm{H}$ patterns.

\section{Mixed Movement Strategies Depending on Both Seed Density and the Interaction with Conspecifics}

We now turn to examining the role of intraspecific interactions among predators. The attraction to feeding conspecifics is known to lead to deviations from the IFD via resource overmatching (see "Plant Recruitment and Predation Patterns"). From a mathematical viewpoint, the effect of conspecifics can be incorporated into the habitat suitability as

$$
f(x, t)=b S(x, t)+c N(x, t)
$$

where $b$ has the usual meaning and the parameter $c$ can be either positive for social foragers or negative for inter-

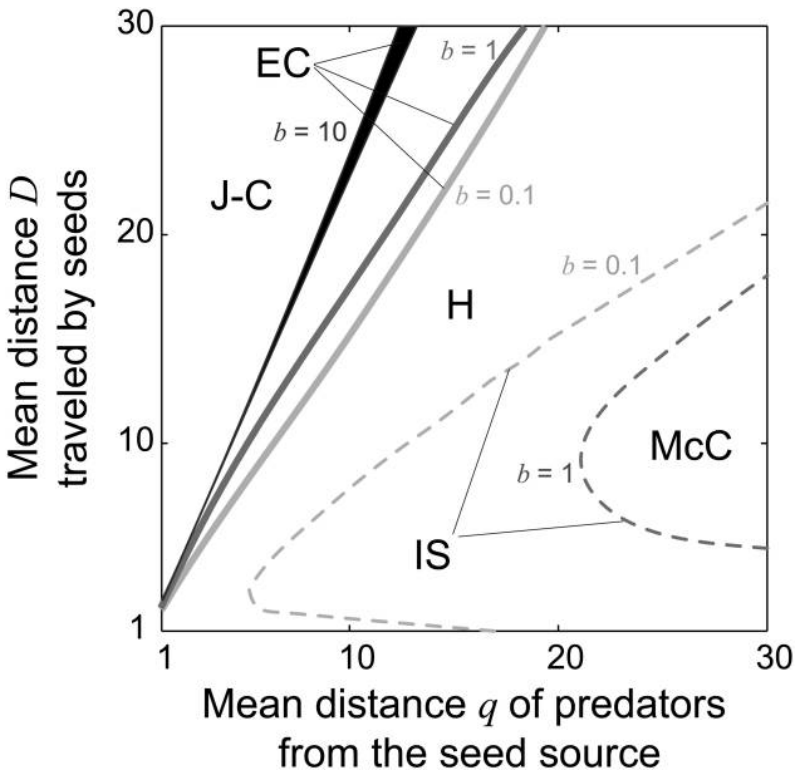

Figure 5: Resulting recruitment patterns in mixed movement strategies of the kind $f(x, t)=b S(x, t)-\tau x$ as functions of the mean distance $q$ of predators from the source and the mean distance $D$ traveled by seeds for different values of the predator's sensitivity $b$ to resource abundance. The exact compensation region (EC; filled) separates the Janzen-Connell $(\mathrm{J}-\mathrm{C})$ pattern from the Hubbell $(\mathrm{H})$ pattern and is generally very small or almost filiform. Dashed lines (technically corresponding to the invariant survival [IS] pattern) separate regions with $\mathrm{H}$ and McCanny $(\mathrm{McC})$ patterns. Line colors indicate different values of the parameter $b$ : light, medium, and dark gray identify $b=0.1, b=1$, and $b=10$, respectively. All parameter values are equal to those specified in figure 2 , except $\tau=1$.

fering foragers. Although different choices have already been described in the literature (see Grünbaum and Veit 2003), a linear relationship between conspecific density and habitat suitability seems to be supported by field data, at least for moderate population densities (Buckley 1996). Stationary solutions can be found via equation (3), which in this case corresponds to the following differential equation

$$
\frac{\partial \bar{S}(x)}{\partial x}=\frac{\left(\frac{\delta}{\varepsilon}-\bar{c}(x)\right) \frac{\partial \Phi(x)}{\partial x}}{b \psi(\bar{S}(x)) \bar{N}(x)+\left(\frac{\delta}{\varepsilon}-c \bar{N}(x)\right)\left[\mu+\bar{N}(x) \frac{\partial \psi(\bar{S}(x))}{\partial \bar{S}(x)}\right]} .
$$

Because the dispersal kernel $\Phi(x)$ does not increase with distance from the source and the functional response $\psi(S)$ does not decrease with seed density, hump-shaped J-C patterns are possible only in the case of social predators $(c>0)$. Interfering foragers $(c<0)$ will always produce 
monotonically decreasing patterns. Notice that this is rigorously true provided that the spatial derivative of the dispersal kernel is not null in the origin. But in any case, even under this circumstance, the result is still valid in any neighborhood of the origin, where the partial derivative of $\Phi(x)$ is negative.

Interestingly, the predator distribution corresponding to a J-C seed recruitment pattern generated by social predators (fig. $3 C$ ) is clumped. In fact, there exists a limit distance within which predators are displaced (fig. 3C, bottom) in a way that is similar to the case of mixed distance- and density-dependent movement strategies (fig. $3 B$ ). This suggests that the clumped predator distributions occurring in J-C patterns may be a general feature and do not depend on a particular movement strategy.

In figure 6 we display the recruitment patterns corresponding to different values of the parameters $q$ and $D$ for different values of the parameter $c$ (i.e., for different sensitivities to conspecific density). We restrict our attention to the case $c>0$ (social foraging), which may result in hump-shaped patterns. Despite the similarity between figures 5 and 6 , there is a principal difference between the two: namely, that the latter lacks a region in the parameter space $(q, D)$ resulting in $\mathrm{McC}$ patterns (at least for the parameter settings we considered). Furthermore, increasing the predators' sensitivity to conspecific abundance (higher $c$ ) leads to a larger region that is characterized by hump-shaped J-C patterns.

\section{Conclusions}

In this article we have introduced a spatially explicit model to describe the effects of different movement strategies of foragers on postdispersal seed predation and plant establishment patterns. The role of postdispersal seed predation per se in shaping plant recruitment patterns is widely recognized in the ecological literature (e.g., Nathan and Muller-Landau 2000) and has also recently been explored via mathematical modeling (Nathan and Casagrandi 2004; Adler and Muller-Landau 2005). However, a deeper mechanistic understanding of this process requires a detailed examination of the behavioral responses of seed predators to the relevant environmental cues. To this end, we have proposed a general formulation of the navigation strategy by which foragers explore their surrounding habitat. This formulation is based on a function that we have called habitat suitability, as in the original work by Fretwell and Lucas (1969). Our model accounts for both IFD and nonIFD seed predators and is flexible enough to reproduce all the basic plant recruitment patterns found in nature (fig. 1). Therefore, it provides the means to link a wide variety of movement strategies to the different vegetation patterns and, in particular, to specify the conditions required for

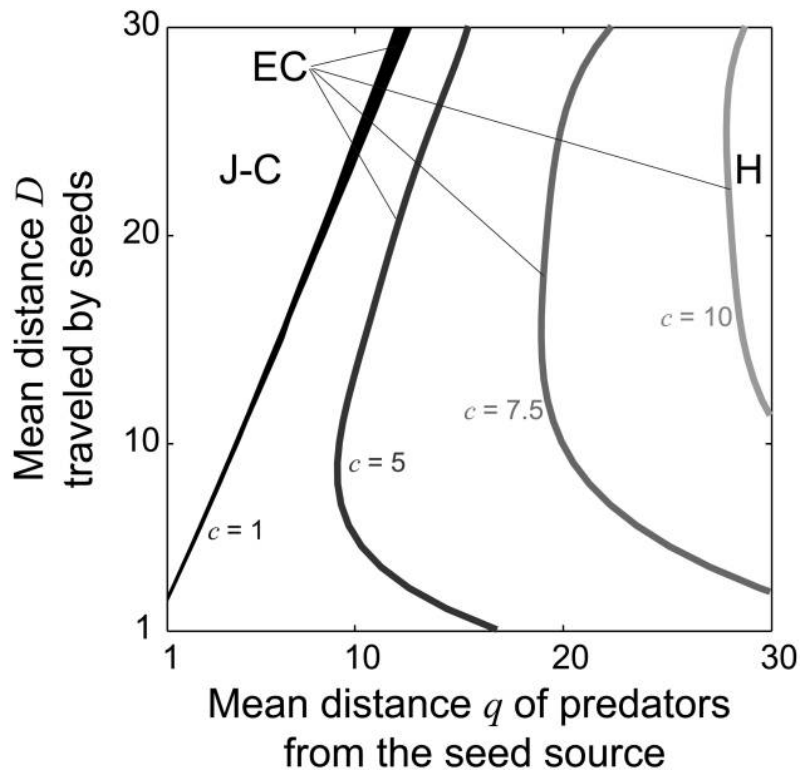

Figure 6: Relationship between the average distance of both dispersal and predation and the corresponding recruitment patterns for different parameter settings of the habitat suitability $f(x, t)=b S(x, t)+c N(x, t)$. See figure 5 for technical details. Parameter values are equal to those specified in figure 2, except $b=1$ and $c=1$ (black), $c=5$ (dark gray), $c=7.5$ (medium gray), and $c=10$ (light gray).

J-C patterns to emerge. This is of special importance because of the hypothesized role of this pattern in the maintenance of species diversity (Janzen 1970; Wright 2002).

Our main finding is that the $\mathrm{J}-\mathrm{C}$ seed recruitment pattern cannot be generated by purely density-responsive seed predators (fig. 2). This result has been obtained under very plausible and general assumptions. Also, postdispersal seed predation exerted by IFD foragers (purely density-responsive foragers with no movement limitations, with perfect knowledge of the resource distribution, and no interference from conspecifics or other organisms) can only result in the EC pattern (fig. 2C). The abundance of IFD foragers sharply declines at some threshold distance from the seed source (fig. 2D). This implies that density-responsive consumers may exhibit patchy distribution even when the resource density gradient is continuous. As IFD seed predators cannot generate a hump-shaped recruitment, J-C patterns represent the most apparent footprint of a deviation from the IFD theory. The two mechanisms tested here that can produce a J-C pattern are attraction to the seed source (distance-dependent predation; fig. $3 A, 3 B$ ) and to conspecifics (social foraging; fig. $3 C$ ). On the other hand, monotonically decreasing patterns (either $\mathrm{H}$ or $\mathrm{McC}$ ) can be found when density-responsive seed foragers have limited navigation capacity (thus, the random component of movement is not negligible; fig. $2 A, 2 B$ ) or, 
in mixed movement strategies, when predators are more sensitive to actual seed abundance than to other cues, provided that the navigation capacity is high (fig. 4). Furthermore, in the case of mixed distance- and densitydependent predation, we have verified that, in J-C patterns, the mean distance of seed foragers from the seed source is smaller than that traveled by dispersed seeds (fig. 5), as already envisaged by Nathan and Casagrandi (2004). As for social foraging, we have shown that the J-C pattern is likely to occur when attraction among conspecifics is large (fig. 6). This result fits well with the experimental finding that socially foraging ants generate J-C patterns, whereas individual foragers do not (Avgar et al. 2008).

The proposed linkage between predators' behavioral traits and vegetation patterns may help to explain the observation that patterns generated by invertebrate seed predators generally conform to the $\mathrm{J}-\mathrm{C}$ model, whereas those generated by vertebrates do not (Hammond and Brown 1998 and references therein). Differences in search tactics and resource selection criteria may be the key to understanding such a disparity: in particular, invertebrates are often distance-responsive, specialized seed predators, whereas vertebrates are typically mobile, generalist consumers (Hammond and Brown 1998; Nathan and Casagrandi 2004). Furthermore, many invertebrates behave as social foragers and make large use of chemosensory cues so as to efficiently share information about resource availability and distribution (e.g., van der Goes van Naters and Carlson 2006). Therefore, our findings provide strong theoretical support for a distinction between the outcomes of seed predation exerted by either vertebrate or invertebrate consumers on the basis of their different foraging strategies.

Our conclusions are expected to be robust because they do not depend on particular functional forms or peculiar parameter settings. Also, all the simulations presented in this article are representative of the behavior of the system for a wide range of conditions. Moreover, the model parameters are easy to interpret and the results are amenable to comparison with empirical data. However, a specific parameterization of the model might not be so easy. Although most of the involved quantities can actually be derived from field experiments, the estimation of others may prove to be difficult.

In particular, dispersal kernels can be fitted with inverse statistical methods (Ribbens et al. 1994; Clark et al. 1998, 1999), mechanistic and phenomenological models (Nathan et al. 2001; Katul et al. 2005), or genetic techniques (González-Martínez et al. 2006), whereas predators' functional responses can be derived from exclosures studies (see Hulme and Hunt 1999 with reference to seed predation; see also Nathan and Casagrandi 2005). As for diffusion coefficients, several estimation techniques have been proposed, ranging from the fitting of the resulting normal distribution to the tracking of individual organisms and the computation of population fluxes (as reviewed in Turchin 1998; Okubo and Levin 2001). Berg (1993) and Murray (2004) provide useful historical framings of the topic, as well as comprehensive discussions of the typical difficulties arising in this context.

In contrast, measuring the directed component of movement (given by the product between the taxis coefficient and the habitat suitability function) represents a difficult challenge, as one would need to resort to suitable "cafeteria-style" experiments (e.g., Busher 1996; Meiners and Stiles 1997). For instance, the interplay between density- and distance-dependent seed predation could be studied via field trials in which seed predators have to forage over food patches with (i) different resource densities and the same distance from the point of release of predators or (ii) the same resource density and different distances from the point of release of predators. As for social foraging, statistical techniques aimed at assessing the relative importance of resource and conspecific abundances in orienting the predators' movement have recently been proposed in the literature (Grünbaum and Veit 2003).

Extensions of the model are possible in order to add further realism and incorporate new features. In particular, the model could be easily cast in a 2D space and modified to account for seed predators whose focal place of activity is not the seed source (as in the experimental setting used by Avgar et al. [2008]). The study of these extensions together with the analysis of landscapes with multiple seed sources are important steps that are being explored (L. Mari, R. Casagrandi, and M. Gatto, unpublished manuscript). More realistic and complicated assumptions could be used for the formulation of the habitat suitability function and the description of how consumers actually assess the habitat suitability itself (e.g., to describe predators with complex social interactions). The model presented here is a first step toward a better description and understanding of the processes that lead to plant recruitment. Hopefully, this will yield even greater insight into the mechanisms involved in the crucial problem of biodiversity maintenance.

\section{Acknowledgments}

Part of the work for this article was performed while L.M. was visiting S. A. Levin's laboratory at Princeton University (Princeton, NJ; project "Internazionalizzazione del Sistema Universitario II O4 CE 4968”) and while R.C. was at the Institute for Advanced Studies at the Hebrew University of Jerusalem. R.N. and T.A. acknowledge support from the International Arid Land Consortium (IALC 03R/25) and from the Israel Science Foundation (ISF-474/02 and 
ISF-FIRST-1316/05). R.N. also acknowledges support from the U.S. National Science Foundation (NSF-DEB0453665), the Simon and Ethel Flegg Fellowship, and the Friedrich Wilhelm Bessel Research Award of the Humboldt Foundation. We wish to thank two anonymous reviewers for improving the quality of the article.

\section{Literature Cited}

Abrahams, M. V. 1986. Patch choice under perceptual constraints: a cause for departures from an ideal free distribution. Behavioral Ecology and Sociobiology 19:409-415.

Adler, F. R., and H. C. Muller-Landau. 2005. When do localized natural enemies increase species richness? Ecology Letters 8:438447.

Andersson, M. 1981. Central place foraging in the whinchat (Saxicola rubetra). Ecology 62:528-544.

Armsworth, P. R., and J. E. Roughgarden. 2005. The impact of directed versus random movement on population dynamics and biodiversity patterns. American Naturalist 165:449-465.

Augspurger, C. K. 1983a. Offspring recruitment around tropical trees: changes in cohort distance with time. Oikos 40:189-196.

- 1983b. Seed dispersal of the tropical tree, Platypodium elegans, and the escape of its seedlings from fungal pathogens. Journal of Ecology 71:759-771.

- 1984. Seedling survival of tropical tree species: interactions of dispersal distance, light-gaps, and pathogens. Ecology 65:17051712 .

Augspurger, C. K., and K. Kitajima. 1992. Experimental studies of seedling recruitment from contrasting seed distributions. Ecology 73:1270-1284.

Avgar, T., I. Giladi, and R. Nathan. 2008. Linking traits of foraging animals to spatial patterns of plants: social and solitary ants generate opposing patterns of surviving seeds. Ecology Letters 11:224234.

Barone, J. A. 2000. Comparison of herbivores and herbivory in the canopy and understory for two tropical tree species. Biotropica 32:307-317.

Barot, S., G. Ginoux, and J. Menaut. 1999. Seed shadow, survival and recruitment: how simple mechanisms lead to dynamics of population recruitment curves. Oikos 86:320-330.

Bautista, L. M., J. C. Alonso, and J. A. Alonso. 1995. A field test of ideal free distribution in flock-feeding common cranes. Journal of Animal Ecology 64:747-757.

Beauchamp, G., M. Belisle, and L.-A. Giraldeau. 1997. Influence of conspecific attraction on the spatial distribution of learning foragers in a patchy habitat. Iournal of Animal Ecology 66:671-682.

Berg, H. C. 1993. Random walks in biology. Princeton University Press, Princeton, NJ.

Bonanomi, G., M. Rietkerk, S. C. Dekker, and S. Mazzoleni. 2008. Islands of fertility induce co-occurring negative and positive plantsoil feedbacks promoting coexistence. Plant Ecology 197:207-218.

Boudreau, S., and M. J. Lawes. 2008. Density- and distance-dependent seedling survival in a ballistically dispersed subtropical tree species Philenoptera sutherlandii. Journal of Tropical Ecology 24: $1-8$.

Buckley, N. J. 1996. Food finding and the influence of information, local enhancement, and communal roosting on foraging success of North American vultures. Auk 113:473-488.
1997. Spatial-concentration effects and the importance of local enhancement in the evolution of colonial breeding in seabirds. American Naturalist 149:1091-1112.

Burkey, T. V. 1994. Tropical tree species diversity: a test of the JanzenConnell model. Oecologia (Berlin) 97:533-540.

Busher, P. E. 1996. Food caching behavior of beavers (Castor canadensis): selection and use of woody species. American Midland Naturalist 135:343-348.

Cartar, R. V., and M. V. Abrahams. 1997. Predicting the distribution of organisms among a few patches: problems with detecting departures from the ideal free distribution. Oikos 78:388-393.

Clark, D. A., and D. B. Clark. 1984. Spacing dynamics of a tropical rain forest tree: evaluation of the Janzen-Connell model. American Naturalist 124:769-788.

Clark, J. S., E. Macklin, and L. Wood. 1998. Stages and spatial scales of recruitment limitation in southern Appalachian forests. Ecological Monographs 68:213-235.

Clark, J. S., M. Silman, R. Kern, E. Macklin, and J. HilleRisLambers. 1999. Seed dispersal near and far: patterns across temperate and tropical forests. Ecology 80:1475-1494.

Clode, D. 1993. Colonially breeding seabirds: predators or prey? Trends in Ecology \& Evolution 8:336-338.

Condit, R., S. P. Hubbell, and R. B. Foster. 1992. Recruitment near conspecific adults and the maintenance of tree and shrub diversity in a Neotropical forest. American Naturalist 140:261-286.

Connell, J. H. 1971. On the role of natural enemies in preventing competitive exclusion in some marine animals and in rain forest trees. Pages 298-312 in P. J. D. Boer and G. R. Gradwel, eds. Dynamics of populations. Pudoc Scientific, Wageningen, Netherlands.

Connell, J. H., J. G. Tracey, and L. J. Webb. 1984. Compensatory recruitment, growth, and mortality as factors maintaining rain forest tree diversity. Ecological Monographs 54:141-164.

Cosner, C. 2005. A dynamic model for the ideal-free distribution as a partial differential equation. Theoretical Population Biology 67: 101-108

Denslow, J. S. 1980. Notes on the seedling ecology of a large-seeded species of Bombacaceae. Biotropica 12:220-222.

De Steven, D., and F. E. Putz. 1984. Impact of mammals on early recruitment of a tropical canopy tree, Dipteryx panamensis, in Panama. Oikos 43:207-216.

Dwyer, G., and W. F. Morris. 2006. Resource-dependent dispersal and the speed of biological invasions. American Naturalist 167: 165-176.

Farnsworth, K. D., and A. R. A. Anderson. 2001. How simple grazing rules can lead to persistent boundaries in vegetation communities. Oikos 95:15-24.

Flaxman, S. M., and H. K. Reeve. 2006. Putting competition strategies into ideal free distribution models: habitat selection as a tug of war. Journal of Theoretical Biology 243:587-593.

Fleming, T. H., and E. R. Heithaus. 1981. Frugivorous bats, seed shadows, and the structure of tropical forests. Biotropica 13:4553.

Forget, P. 1989. La régénération naturelle d'une espèce autochore de la Forêt Guyanaise: Eperua falcata Aublet (Caesalpiniaceae). Biotropica 21:115-125.

- 1992. Regeneration ecology of Eperua grandiflora (Caesalpiniaceae), a large-seeded tree in French Guiana. Biotropica 24: $146-156$.

1993. Post-dispersal predation and scatterhoarding of Dip- 
teryx panamensis (Papilionaceae) seeds by rodents in Panama. Oecologia (Berlin) 94:225-261.

. 1994. Recruitment pattern of Vouacapoua americana (Caesalpiniaceae), a rodent-dispersed tree species in French Guiana. Biotropica 26:408-419.

Forget, P., E. Munoz, and E. Giles Leigh Jr. 1994. Predation by rodents and bruchid beetles on seeds of Schelea palms on Barro Colorado Island, Panama. Biotropica 26:420-426.

Fragoso, J. M. V. 1997. Tapir-generated seed shadows: scale-dependent patchiness in the Amazon rain forest. Journal of Ecology 85: 519-529.

Freckleton, R. P., and O. T. Lewis. 2006. Pathogens, density dependence and the coexistence of tropical trees. Proceedings of the Roval Society B: Biological Sciences 273:2909-2916.

Fretwell, S. D., and H. L. Lucas. 1969. On territorial behavior and other factors influencing habitat distribution in birds. I. Theoretical development. Acta Biotheoretica 19:16-36.

Gilbert, G. S., and D. De Steven. 1996. A canker disease of seedlings and saplings of Tetragastris panamensis (Burseraceae) caused by Botryosphaeria dothidea in a lowland tropical forest. Plant Disease 80:684-687.

Gilbert, G. S., K. E. Harms, D. N. Hamill, and S. P. Hubbell. 2001. Effects of seedling size, El Niño drought, seedling density, and distance to nearest conspecific adult on 6-year survival of Ocotea whitei seedlings in Panamá. Oecologia (Berlin) 127:509-516.

González-Martínez, S. C., J. Burczyk, R. Nathan, N. Nanos, L. Gil, and R. Alía. 2006. Effective gene dispersal and female reproductive success in Mediterranean maritime pine (Pinus pinaster Aiton). Molecular Ecology 15:4577-4588.

Goulson, D., S. A. Hawshon, and J. C. Stout. 1998. Foraging bumble bees avoid flowers already visited by conspecifics or by other bumble bee species. Animal Behaviour 55:199-206.

Grau, H. R. 2000. Regeneration patterns of Cedrela lilloi (Meliaceae) in northwestern Argentina subtropical montane forests. Iournal of Tropical Ecology 16:227-242.

Gray, R. D., and M. Kennedy. 1994. Perceptual constraints on optimal foraging: a reason for departures from the ideal free distribution. Animal Behaviour 47:469-471.

Grünbaum, D. 1999. Advection-diffusion equations for generalized tactic searching behaviors. Journal of Mathematical Biology 38: 169-194.

Grünbaum, D., and R. Veit. 2003. Black-browed albatrosses foraging on antarctic krill: density-dependence through local enhancement? Ecology 84:3265-3275.

Guittet, J., and J.-C. Laberche. 1974. L'implantation du pin sylvestre sur pelouse xérophile en forêt de Fontainebleu. II. Demographie des graines et des plantules au voisinage des vieux arbres. Acta Oecologica-Oecologia Plantarum 9:111-130.

Hakoyama, H. 2003. The ideal free distribution when the resource is variable. Behavioral Ecology 14:109-115.

Hammond, D. S., and V. K. Brown. 1998. Disturbance, phenology and life-history characteristics: factors influencing distance/density-dependent attack on tropical seeds and seedlings. Pages 5178 in D. M. Newbery, H. H. T. Prins, and N. D. Brown, eds. Dynamics of tropical communities. Blackwell, Oxford.

Hancock, P. A., E. J. Milner-Gulland, and M. J. Keeling. 2006. Modelling the many-wrongs principle: the navigational advantages of aggregation in nomadic foragers. Journal of Theoretical Biology 240:302-310

Harms, K. E., S. J. Wright, O. Calderón, A. Hernández, and E. Allen
Herre. 2000. Pervasive density-dependent recruitment enhances seedling diversity in a tropical forest. Nature 404:493-495.

Hart, T. B. 1995. Seed, seedling and sub-canopy survival in monodominant and mixed forests of the Ituri Forest, Africa. Journal of Tropical Ecology 11:443-459.

Hassell, M. P., and R. M. May. 1974. Aggregation of predators and insect parasites and its effect on stability. Journal of Animal Ecology 43:567-594.

Hölldobler, B., and E. O. Wilson. 1990. The ants. Belknap, Cambridge, MA.

Holling, C. S. 1959. Some characteristics of simple types of predation and parasitism. Canadian Entomologist 91:385-398.

Holmes, E. E., M. A. Lewis, J. E. Banks, and R. R. Veit. 1994. Partial differential equations in ecology: spatial interactions and population dynamics. Ecology 75:17-29.

Holyoak, M., R. Casagrandi, R. Nathan, E. Revilla, and O. Spiegel. 2008. Trends and missing parts in the study of movement ecology. Proceedings of the National Academy of Sciences of the USA (forthcoming).

Howe, H. F. 1993. Aspects of variation in a Neotropical seed dispersal system. Vegetatio 107/108:149-162.

Howe, H. F., and R. B. Primack. 1975. Differential seed dispersal by birds of the tree Casearia nitida (Flacourtiaceae). Biotropica 7: 278-283.

Howe, H. F., and J. Smallwood. 1982. Ecology of seed dispersal. Annual Review of Ecology and Systematics 13:201-228.

Howe, H. F., E. W. Schupp, and L. C. Westley. 1985. Early consequences of seed dispersal for a Neotropical tree (Virola surinamensis). Ecology 66:781-791.

Hubbell, S. P. 1979. Tree dispersion, abundance, and diversity in a tropical dry forest. Science 203:1299-1309.

- 1980. Seed predation and the coexistence of tree species in tropical forests. Oikos 35:214-229.

Hubbell, S. P., R. Condit, R. B. Foster, P. J. Grubb, and C. D. Thomas. 1990. Presence and absence of density dependence in a Neotropical tree community. Philosophical Transactions of the Royal Society B: Biological Sciences 330:269-281.

Hugie, D. M., and T. C. Grand. 1998. Movement between patches, unequal competitors and the ideal free distribution. Evolutionary Ecology 12:1-19.

Hulme, P. E., and M. K. Hunt. 1999. Rodent post-dispersal seed predation in deciduous woodland: predator response to absolute and relative abundance of prey. Journal of Animal Ecology 68: 417-428.

Hyatt, L. A., M. S. Rosenberg, T. G. Howard, G. Bole, W. Fang, J. Anastasia, K. Brown, et al. 2003. The distance dependence prediction of the Janzen-Connell hypothesis: a meta-analysis. Oikos 103:590-602.

Itoh, A., T. Yamakura, K. Ogino, H. Seng Lee, and P. S. Ashton. 1997. Spatial distribution patterns of two predominant emergent trees in a tropical rainforest in Sarawak, Malaysia. Plant Ecology 132: 121-136.

Jackson, A. L., S. Humphries, and G. D. Ruxton. 2004. Resolving the departures of observed results from the ideal free distribution with simple random movements. Journal of Animal Ecology 73: 612-622.

Janzen, D. H. 1970. Herbivores and the number of tree species in tropical forests. American Naturalist 104:501-528.

- 1971a. Escape of juvenile Dioclea megacarpa (Leguminosae) 
vines from predators in a deciduous tropical forest. American Naturalist 105:97-112.

. 1971b. Seed predation by animals. Annual Review of Ecology and Systematics 2:465-492.

. 1972. Escape in space by Sterculia apetala seeds from the bug Dysdercus fasciatus in a Costa Rican deciduous forest. Ecology 53:350-361.

- 1976. Why bamboos wait so long to flower. Annual Review of Ecology and Systematics 7:347-391.

Janzen, D. H., G. A. Miller, J. Hackforth-Jones, C. M. Pond, K. Hooper, and D. P. Janos. 1976. Two Costa Rican bat-generated seed shadows of Andira inermis (Leguminosae). Ecology 57:10681075.

Kacelnik, A., J. R. Krebs, and C. Bernstein. 1992. The ideal free distribution and predator-prey populations. Trends in Ecology \& Evolution 7:50-55.

Kareiva, P., and G. Odell. 1987. Swarms of predators exhibit "preytaxis" if individual predators use area-restricted search. American Naturalist 130:233-270.

Katul, G. G., A. Porporato, R. Nathan, M. Siqueira, M. B. Soons, D. Poggi, H. S. Horn, and S. A. Levin. 2005. Mechanistic analytical models for long-distance seed dispersal by wind. American Naturalist 166:368-381.

Kitajima, K., and C. K. Augspurger. 1989. Seed and seedling ecology of a monocarpic tropical tree, Tachigalia versicolor. Ecology 70: $1102-1114$

Koops, M. A., and M. V. Abrahams. 2003. Integrating the roles of information and competitive ability on the spatial distribution of social foragers. American Naturalist 161:586-600.

Levin, S. A., H. C. Muller-Landau, R. Nathan, and J. Chave. 2003. The ecology and evolution of seed dispersal: a theoretical perspective. Annual Review of Ecology, Evolution, and Svstematics 34:575-604.

Mazzoleni, S., G. Bonanomi, F. Giannino, M. Rietkerk, S. C. Dekker, and F. Zucconi. 2007. Is plant biodiversity driven by decomposition processes? an emerging new theory on plant diversity. Community Ecology 8:103-109.

McCanny, S. J. 1985. Alternatives in parent-offspring relationships in plants. Oikos 45:148-149.

McCanny, S. J., and P. B. Cavers. 1987. The escape hypothesis: a test involving a temperate, annual grass. Oikos 49:67-76.

Meiners, S. J., and E. W. Stiles. 1997. Selective predation on the seeds of woody plants. Journal of the Torrey Botanical Society 124:67-70.

Moorcroft, P. R., S. W. Pacala, and M. A. Lewis. 2006. Potential role of natural enemies during tree range expansions following climate change. Journal of Theoretical Biology 241:601-616.

Muller-Landau, H. C., S. J. Wright, O. Calderón, S. P. Hubbell, and R. B. Foster. 2002. Assessing recruitment limitation: concepts, methods and case-studies from a tropical forest. Pages 35-53 in D. J. Levey, W. R. Silva, and M. Galetti, eds. Seed dispersal and frugivory: ecology, evolution and conservation. CABI, Wallingford, UK.

Muller-Landau, H. C., S. A. Levin, and J. E. Keymer. 2003. Theoretical perspectives on evolution of long-distance dispersal and the example of specialized pests. Ecology 84:1957-1967.

Murray, J. D. 2004. Mathematical biology II: spatial models and biomedical applications. Springer, New York.

Naef-Daenzer, B. 2000. Patch time allocation and patch sampling by foraging great and blue tits. Animal Behaviour 59:989-999.

Nathan, R., and R. Casagrandi. 2004. A simple mechanistic model of seed dispersal, predation and plant establishment: JanzenConnell and beyond. Journal of Ecology 92:733-746.

- 2005. A simple mechanistic model of seed dispersal, predation and plant establishment: Janzen-Connell and beyond (erratum). Journal of Ecology 93:852.

Nathan, R., and H. C. Muller-Landau. 2000. Spatial patterns of seed dispersal, their determinants and consequences for recruitment. Trends in Ecology \& Evolution 15:278-285.

Nathan, R., U. N. Safriel, I. Noy-Meir, and G. Schiller. 2000. Spatiotemporal variation in seed dispersal and recruitment near and far from Pinus halepensis trees. Ecology 81:2156-2169.

Nathan, R., U. N. Safriel, and I. Noy-Meir. 2001. Field validation and sensitivity analysis of a mechanistic model for tree seed dispersal by wind. Ecology 82:374-388.

Nathan, R., W. M. Getz, E. Revilla, M. Holyoak, R. Kadmon, D. Saltz, and P. E. Smouse. 2008. A movement ecology paradigm for unifying organismal movement research. Proceedings of the National Academy of Sciences of the USA (forthcoming).

Nemeth, E., P. Bossew, and C. Plutzar. 2005. A distance-dependent estimation of foraging ranges of neighbouring bird colonies. Ecological Modelling 182:67-73.

Norghauer, J., J. R. Malcolm, B. L. Zimmerman, and J. M. Felfili. 2006. An experimental test of density- and distant-dependent recruitment of mahogany (Swietenia macrophylla) in southeastern Amazonia. Oecologia (Berlin) 148:437-446.

Notman, E., D. L. Gorchov, and F. Cornejo. 1996. Effect of distance, aggregation, and habitat on levels of seed predation for two mammal-dispersed Neotropical rain forest tree species. Oecologia (Berlin) 106:221-227.

Okubo, A. 1980. Diffusion and ecological problems: mathematical models. Springer, Berlin.

Okubo, A., and S. A. Levin. 2001. Diffusion and ecological problems. Springer, New York.

Okuda, T., N. Kachi, S. K. Yap, and N. Manokaran. 1997. Tree distribution pattern and fate of juveniles in a lowland tropical rain forest implications for regeneration and maintenance of species diversity. Plant Ecology 131:155-171.

Packer, A., and K. Clay. 2000. Soil pathogens and spatial patterns of seedling mortality in a temperate tree. Nature 404:278-281.

Patlak, C. S. 1953. Random walk with persistence and external bias. Bulletin of Mathematical Biophysics 15:311-338.

Pöysä, H. 1992. Group foraging in patchy environments: the importance of coarse-level local enhancement. Ornis Scandinavica 23:159-166.

Prince, P. J., and J. R. Dorman. 1981. High order embedded RungeKutta formulae. Journal of Computational and Applied Mathematics 7:67-75.

Ramirez, N., and M. K. Arroyo. 1987. Variación espacial y temporal en la depredación de semillas de Copaifera publiflora Benth. (Leguminosae: Caesalpinioideae) en Venezuela. Biotropica 19:32-39.

Ribbens, E., J. A. Silander Jr., and S. W. Pacala. 1994. Seedling recruitment in forests: calibrating models to predict patterns of tree seedling dispersion. Ecology 75:1794-1806.

Rosenberg, D. K., and K. S. McKelvey. 1999. Estimation of habitat selection for central place foraging animals. Journal of Wildlife Management 63:1028-1038.

Ruxton, G. D., and P. Rohani. 1999. Fitness-dependent dispersal in metapopulations and its consequences for persistence and synchrony. Journal of Animal Ecology 67:643-660. 
Schupp, E. W. 1988. Factors affecting post-dispersal seed survival in a tropical forest. Oecologia (Berlin) 76:525-530.

Schupp, E. W., and M. Fuentes. 1995. Spatial patterns of seed dispersal and the unification of plant population ecology. Ecoscience 2:267-275.

Segel, L. A. 1977. A theoretical study of receptor mechanisms in bacterial chemotaxis. SIAM Journal of Applied Mathematics 32: 653-665.

Serrano, J. M., J. A. Delgado, F. Lopez, F. J. Acosta, and S. G. Fungairino. 2001. Multiple infestation by seed predators: the effect of loculate fruits on intraspecific insect larval competition. Acta Oecologica-International Journal of Ecology 22:153-160.

Shochat, E., Z. Abramsky, B. Pinshow, and M. A. E. Whitehouse. 2002. Density-dependent habitat selection in migratory passerines during stopover: what causes the deviation from IFD? Evolutionary Ecology 16:469-488.

Skellam, J. G. 1951. Random dispersal in theoretical populations. Biometrika 38:196-218.

Slaa, E. J., J. Wassenberg, and J. C. Biesmeijer. 2003. The use of fieldbased social information in eusocial foragers: local enhancement among nestmates and heterospecifics in stingless bees. Ecological Entomology 28:369-379.

Sork, V. L. 1987. Effects of predation and light on seedling establishment in Gustavia superba. Ecology 68:1341-1350.

Stephens, D. W., and J. R. Krebs. 1986. Foraging theory. Princeton University Press, Princeton, NJ.

Sutherland, W. J. 1983. Aggregation and the ideal free distribution. Journal of Animal Ecology 52:821-828.

Sutherland, W. J., J. A. Gill, and K. Norris. 2002. Density-dependent dispersal in animals: concepts, evidence, mechanisms and consequences. Pages 134-151 in J. M. Bullock, R. E. Kenward, and R. S. Hails, eds. Dispersal ecology. Blackwell, Malden, MA.

Takeuchi, Y., and T. Nakashizuka. 2007. Effect of distance and density on seed/seedling fate of two dipterocarp species. Forest Ecology and Management 247:167-174.

Terborgh, J., and S. J. Wright. 1994. Effects of mammalian herbivores on plant recruitment in two Neotropical forests. Ecology 75:18291833.

Thompson, D. B., J. H. Brown, and W. D. Spencer. 1991. Indirect facilitation of granivorous birds by desert rodents: experimental evidence from foraging patterns. Ecology 72:852-863.

Traveset, A. 1990. Post-dispersal predation of Acacia farnesiana seeds by Stator vachelliae (Bruchidae) in Central America. Oecologia (Berlin) 84:506-512.
Turchin, P. 1998. Quantitative analysis of movement. Sinauer, Sunderland, MA.

Turchin, P., and P. Kareiva. 1989. Aggregation in Aphis varians: an effective strategy for reducing predation risk. Ecology 70:10081011.

Turner, E. R. A. 1964. Social feeding in birds. Behaviour 24:1-46.

Utne, A. C. W., D. L. Aksnes, and J. Giske. 1993. Food, predation risk and shelter: an experimental study on the distribution of adult two-spotted goby Gobiusculus flavescens (Fabricius). Journal of Experimental Marine Biology and Ecology 166:203-216.

van der Goes van Naters, W., and J. R. Carlson. 2006. Insects as chemosensors of humans and crops. Nature 444:302-307.

Vergassola, M., E. Villermaux, and B. I. Shraiman. 2007. Infotaxis as a strategy for searching without gradients. Nature 445:406-409.

Wenny, D. G. 2000. Seed dispersal, seed predation, and seedling recruitment of a Neotropical montane tree. Ecological Monographs 70:331-351.

Wilby, A., and M. Shachak. 2004. Shrubs, granivores and annual plant community stability in an arid ecosystem. Oikos 106:209216.

Wills, C., R. Condit, R. B. Foster, and S. H. Hubbell. 1997. Strong density- and diversity-related effects help to maintain tree species diversity in a Neotropical forest. Proceedings of the National Academv of Sciences of the USA 94:1252-1257.

Wills, C., K. E. Harms, R. Condit, D. King, J. Thompson, F. He, H. C. Muller-Landau, et al. 2006. Nonrandom processes maintain diversity in tropical forests. Science 311:527-531.

Willson, M. F. 1993. Dispersal mode, seed shadows, and colonization patterns. Vegetatio 107/108:261-280.

Willson, M. F., and A. Traveset. 2000. The ecology of seed dispersal. Pages 85-110 in M. Fenner, ed. Seeds: the ecology of regeneration in plant communities. CAB International, Wallingford.

Wilson, D. E., and D. H. Janzen. 1972. Predation on Scheelea palm seeds by bruchid beetles: seed density and distance from the parent palm. Ecology 53:954-959.

Wright, S. J. 1983. The dispersion of eggs by a bruchid beetle among Scheelea palm seeds and the effect of distance to the parent palm. Ecology 64:1016-1021.

- 2002. Plant diversity in tropical forests: a review of mechanisms of species coexistence. Oecologia (Berlin) 130:1-14.

Wyatt, J., and M. R. Silman. 2004. Distance-dependence in two Amazonian palms: effects of spatial and temporal variation in seed predator communities. Oecologia (Berlin) 140:26-35.

Associate Editor: Marc Mangel Editor: Donald L. DeAngelis 